\title{
Isolation and characterization of acid and pepsin soluble collagen extracted from sharpnose stingray (Dasyatis zugei) skin
}

\author{
Ong, T.Y., Shaik, M.I. and *Sarbon, N.M. \\ Faculty of Fishery and Food Science, Universiti Malaysia Terengganu (UMT), 21030 Kuala Nerus, \\ Terengganu, Malaysia.
}

\author{
Article history: \\ Received: 1 July 2020 \\ Received in revised form: \\ 113 August 2020 \\ Accepted: 14 September 2020 \\ Available Online: 20 \\ December 2020 \\ Keywords: \\ Acid Soluble Collagen \\ (ASC), \\ Pepsin Soluble Collagen \\ (PSC), \\ Sharpnose stingray (Dasyatis \\ zugei), \\ Fish collagen, \\ Collagen extraction
}

DOI:

https://doi.org/10.26656/fr.2017.5(3).322

\section{Introduction}

The origin of the word 'collagen' is from a Greek term, 'kola' which has a definition of gum/glue and 'gen' which means producing. Collagen can be extracted from various tissues, such as tendons, ligaments, and other connective tissues (Silvipriya et al., 2015). Traditionally, commercialized collagens have been mainly obtained through the slaughter of land-based animals such as pigs and cows (Pal and Suresh, 2017). However, recent years have seen the proliferation of infectious diseases such as bovine spongiform encephalopathy (BSE), transmissible spongiform encephalopathy (TSE), foot-and-mouth disease (FMD) and avian influenza (Baderi and Sarbon, 2019). Thus, recent studies have emphasized on the extraction of collagen from marine organisms to replace bovine and porcine collagen (El-Rashidy et al., 2015). Collagen can be extracted through a series of complex procedures, beginning with the collection of raw materials, pretreatment, extraction, purification and some more steps before to be used in different industries. On the basis of the extraction methods, collagens can extract by four distinct methods which are salting-out method, alkali method, acid method and enzyme method (Schmidt et al., 2016).
The two most common extraction methods are acid hydrolysis for the extraction of acid soluble collagen (ASC) and enzymatic hydrolysis for the extraction of pepsin soluble collagen (PSC) (Schmidt et al., 2016). In the extraction of acid soluble collagen (ASC), both organic acids (acetic acid, citric acid, lactic acid) and inorganic acids (hydrochloric acid, phosphoric acid) can be used. One of the very ideal methods of extracting collagen protein is through the use of an enzyme. The enzyme has greater reaction selectivity and is less harmful to the collagen protein. In the extraction of enzyme soluble collagen, enzymes such as pepsin, trypsin, papain, Novozymes, Alcalase $\AA$ and Flavourzyme can be used (Schmidt et al., 2016). It is reported that collagen extracted using pepsin had a significantly higher yield than acid-extracted collagen. In addition, enzymatic methods produce less waste and can reduce processing time, but are more costly (Schmidt et al., 2016).

Characterization of the physicochemical properties of the extracted collagens is necessary in order to determine their suitability as alternative collagen sources for application in various industries. In terms of chemical properties such as chemical composition, protein 
concentration, amino acid composition and zeta potential of collagens were analyzed (Wang et al., 2009; Kaewdang et al., 2014). Physical properties such as thermal stability, functional group, molecular weight, solubility, viscosity and morphology of alternative collagens were also characterized (El-Rashidy et al., 2015; Hukmi and Sarbon, 2017; Hamdan and Sarbon, 2019).

Stingray is a cartilaginous fish with dorsoventrally flattened bodies and their gill slits were located ventrally on the underside of their body. Of the 10 distinctive types of stingray, sharpnose stingray (Dasyatis zugei) is a common species found within Malaysian oceans (Lim et al., 2014). Unique features of D. zugei including its elongated and pointed snout, broadly rounded disc, presence of a row of small thorns on its tail before sting in adults, short, tapering tail without bands allowing researchers to differentiate them (Matsunuma et al., 2011). However, studies on stingrays remain limited in terms of their nutritional composition. To make more effective use of the wastes generated during fish processing, therefore this study aims to isolate and characterize acid-solubilized collagen (ASC) and pepsin solubilized collagen (PSC) from the skin of the sharpnose stingray.

\section{Materials and methods}

\subsection{Materials}

Sharpnose stingrays (Dasyatis zugei) were obtained from local market in Marang, Terengganu, Malaysia. The skins were removed manually and washed before storage at $-80^{\circ} \mathrm{C}$ for further use. Enzyme pepsin from the source of porcine gastric mucosa used was purchased from Sigma-Aldrich (M) Sdn. Bhd. Selangor, Malaysia. The chemicals used in the extraction and analysis of the collagen were of analytical grade. A marine source of commercial collagen type I from tilapia scale was purchased from Personal Formula Resources Sdn. Bhd. in Selangor, Malaysia.

\subsection{Pre-treatment}

To remove non-collagenous proteins, the samples were soaked and stirred in $0.1 \mathrm{M}$ sodium hydroxide $(\mathrm{NaOH})$ solution with a ratio of 1:8 (w/v) for 6 hrs. The $\mathrm{NaOH}$ solutions were changed every $3 \mathrm{hrs}$ and the samples were then washed thoroughly with cold distilled water until the rinsed water become neutral as measured using a pH meter (Baderi and Sarbon, 2019). The whole procedure was carried out at a temperature of $4^{\circ} \mathrm{C}$. The treated skins were soaked in $0.5 \mathrm{M}$ of EDTA at $\mathrm{pH} 7.4$ with a ratio of $1: 8(\mathrm{w} / \mathrm{v})$ for $24 \mathrm{hrs}$ with continuous stirring to remove any mineral presence. The solutions were changed every 12 hrs. The samples were then washed in distilled water 3 times before proceeding with the extraction process (Baderi and Sarbon, 2019).

\subsection{Isolation of acid soluble collagen (ASC)}

The extraction of the acid soluble collagen (ASC) was conducted following the method of Kaewdang et al. (2014), with slight modification. First, each pre-treated sample was extracted using $0.5 \mathrm{M}$ acetic acid with a sample to solution ratio of $1: 50(\mathrm{w} / \mathrm{v})$ for $24 \mathrm{hrs}$ with continuous stirring. Then, the extracts were centrifuged at $9,000 \times \mathrm{g}$ for $30 \mathrm{mins}$ at $4^{\circ} \mathrm{C}$ and the supernatants obtained were separated. The sample residues were reextracted with $0.5 \mathrm{M}$ acetic acid with a sample to solution ratio of $1: 10(\mathrm{w} / \mathrm{v})$ for $24 \mathrm{hrs}$ before being centrifuged again at $9,000 \times \mathrm{g}$ for $30 \mathrm{mins}$ at $4^{\circ} \mathrm{C}$. Both supernatants were combined and sodium chloride $(\mathrm{NaCl})$ was added for the salt-out process until the final concentration of the supernatant was $0.7 \mathrm{M}$ for precipitation to occur. The supernatants were centrifuged again at $2500 \times \mathrm{g}$ for $15 \mathrm{mins}$ in order to obtain the precipitate. The sample was dialyzed (Dialysisbag; Mw1200-1400, MD44-5M, MYM,USA) against distilled water at $4^{\circ} \mathrm{C}$ and then lyophilized. The precipitates were then frozen at $-80^{\circ} \mathrm{C}$ overnight before being freeze-dried.

\subsection{Isolation of pepsin soluble collagen (PSC)}

The extraction of pepsin soluble collagen (PSC) was based on the method of Kaewdang et al. (2014) with slight modification. For the isolation of the pepsin soluble collagen (PSC), a new batch of pre-treated samples were treated with $0.5 \mathrm{M}$ acetic acid which contained $1.5 \%(\mathrm{w} / \mathrm{w})$ pepsin with the sample to solution ratio of $1: 40(\mathrm{w} / \mathrm{v})$ for $30 \mathrm{hrs}$ at $4{ }^{\circ} \mathrm{C}$ with continuous stirring. Then, the extracts were centrifuged at 9,000 $\mathrm{x} g$ for 30 mins at $4^{\circ} \mathrm{C}$ and the supernatants were separated. The residue was re-extracted with $0.5 \mathrm{M}$ acetic acid containing $1.5 \%(\mathrm{w} / \mathrm{w})$ pepsin for $12 \mathrm{hrs}$ before being centrifuged at $9,000 \times \mathrm{g}$ for $30 \mathrm{mins}$ at $4^{\circ} \mathrm{C}$. Both supernatants were combined and sodium chloride $(\mathrm{NaCl})$ was added to salt out until the final concentration of the supernatant reached $0.7 \mathrm{M}$ for precipitation to occur. The supernatant was further centrifuged at $2500 \times \mathrm{x} g$ for 15 mins to obtain the precipitate. The sample was dialyzed (Dialysisbag; Mw1200-1400, MD44-5M, MYM, USA) against distilled water at $4^{\circ} \mathrm{C}$ and then lyophilized. The precipitates were then frozen at $-80^{\circ} \mathrm{C}$ overnight before being freeze-dried. The yields of ASC and PSC were calculated as follows:

$$
\text { Yield of collagen }(\%)=\frac{\text { weight of collagen }}{\text { weight of raw skin (dry weight) }} \times 100
$$


2.5 Chemical composition of raw skin of the sharpnose stingray and isolated collagen

The chemical compositions of the skin of the sharpnose stingray and isolated collagen were analyzed. The chemical compositions, including moisture, protein, fat, and ash content, were determined according to AOAC (2000). The results obtained for the isolated collagens were compared with the commercial collagen.

\subsection{Viscosity analysis}

The viscosity of the extracted collagen was measured using a viscometer (Brookfield DV-1 viscometer (USA)) following the method of Hukmi and Sarbon, (2017). Collagen samples $(0.3 \%)$ were prepared by dissolving 3 $\mathrm{g}$ of lyophilized collagen in $100 \mathrm{~mL}$ of distilled water. The viscosity was measured by placing the solution into the viscometer using spindle No.1 at speed of $100 \mathrm{rpm}$. The collagen solution was heated from $4^{\circ} \mathrm{C}$ to $50^{\circ} \mathrm{C}$ with a heating rate of $4^{\circ} \mathrm{C} / \mathrm{min}$. At each chosen temperature, the solutions were held for 30 mins prior to viscosity measurement. The relative viscosity at each temperature was calculated in comparison to the viscosity of the extracted collagen obtained at $4^{\circ} \mathrm{C}$. Each experiment was performed in triplicate.

\subsection{Thermal stability}

The thermal stabilities of the extracted ASC and PSC were determined following the method performed by Kaewdang et al. (2014) with slight modification. The extracted ASC and PSC in powder form were rehydrated by adding the deionized water at a solid to solution ratio of 1:20 (w/v). The mixtures were allowed to stand under temperature conditions at $4{ }^{\circ} \mathrm{C}$ for 2 days prior to analysis. A thermal property was performed using a differential scanning calorimeter (Perkin Elmer, Norwalk, CT, USA). Calibration was performed using an Indium thermogram. The samples were accurately weighed to $5 \mathrm{mg}$ into aluminium pans and sealed. The samples were scanned at $1{ }^{\circ} \mathrm{C} / \mathrm{min}$ over the range of 20 $50^{\circ} \mathrm{C}$ using iced water as the cooling medium. An empty pan was used as the reference. The maximum transition temperature $\left(T_{\max }\right)$ was estimated from the thermogram obtained. Total denaturation enthalpy $(\Delta \mathrm{H})$ was estimated by measuring the area of the DSC thermogram.

\subsection{Structural properties by Fourier Transform Infrared Spectroscopy (FTIR)}

The extracted ASC and PSC were subject to FTIR analysis in order to determine their structural properties following the method reported by Hukmi and Sarbon (2017), with slight modification. The infrared spectra analysis was used wavenumbers ranging from 4000 to
$400 \mathrm{~cm}^{-1}$ using an infrared spectrophotometer (Nicolet, Thermo Electron, USA). The sample was pre-prepared by mixing the freeze-dried collagen sample with potassium bromide $(\mathrm{KBr})$ at a ratio of 1:30 and moulded into a disc. Automatic transmission signal gain levels were collected for 32 scans at $4 \mathrm{~cm}^{-1}$ resolution against a background spectrum recorded from the clean empty cell at $25^{\circ} \mathrm{C}$. According to the peak of interest at the specific wavenumber and absorbance, the functional group and the mode of its vibration were identified.

\subsection{Solubility}

The solubility of extracted collagens was determined using different $\mathrm{pH}$ levels according to the method developed by Huang et al. (2011) with slight modifications. The lyophilised collagens were dissolved in $0.5 \mathrm{M}$ acetic acid with gentle stirring for $12 \mathrm{hrs}$ to obtain a final concentration of $1 \mathrm{mg} / \mathrm{mL}$. Approximately $8 \mathrm{~mL}$ was transferred to a centrifuge tube and the $\mathrm{pH}$ was then adjusted across the $\mathrm{pH}$ range from 1 to 10 with $6 \mathrm{~N}$ $\mathrm{NaOH}$ or $6 \mathrm{~N} \mathrm{HCl}$. The volume was made up to $10 \mathrm{~mL}$ with distilled water. The solutions were stirred for 30 mins at $4^{\circ} \mathrm{C}$ and centrifuged at $10,000 \mathrm{xg}$ for 30 mins at $4^{\circ} \mathrm{C}$. Protein concentrations in the supernatant were measured by referring to the protein standard curve obtained through Lowry's method (Lowry et al., 1951).

To obtain a standard protein curve, bovine serum albumin (BSA) dilutions were prepared with a concentration range from 0.05 to $1.0 \mathrm{mg} / \mathrm{mL}$. Approximately $0.2 \mathrm{~mL}$ of protein solution for each concentration was mixed with $2 \mathrm{~mL}$ of analytical reagent $(50 \mathrm{~mL}$ of $2 \%$ sodium carbonate, $50 \mathrm{~mL}$ of $0.1 \mathrm{~N}$ sodium hydroxide, $1 \mathrm{~mL}$ of $1.56 \%$ copper sulphate and $1 \mathrm{~mL}$ of $2.37 \%$ sodium potassium tartrate solution). Folin reagent $(0.2 \mathrm{~mL})$ was added into each sample tube and incubated for 30 mins. The absorbance was measured at $570 \mathrm{~nm}$ using UV/Vis spectrophotometer. A standard calibration curve with absorbance against protein concentration was plotted.

The relative solubility (\%) of the sample collagen was determined by comparing the protein concentration at each $\mathrm{pH}$ level to that of the highest protein concentration. The relative solubility of collagen was calculated as follows:

$$
\text { Relative solubility }(\%)=\frac{\text { Protein conc. of supernatant }}{\text { Highest protein conc. }} \times 100
$$

\subsection{Morphological properties}

The microstructures of the extracted collagen were viewed at $1000 \mathrm{x}$ magnification via scanning electron microscopy (SEM) (JOEL JSM-6360 LA, Tokyo, Japan) at an accelerating voltage of $5.0 \mathrm{kV}$. The collagen 
powder was mounted on aluminium cylinder stubs ( 5 $\mathrm{mm} \times 12.5 \mathrm{~mm}$ ) and sputter-coated with Auto fine coater (JOEL JFC 1600, Tokyo, Japan). The samples were observed in a superficial position at 1000x magnification (Hukmi and Sarbon, 2017).

\subsection{Statistical analysis}

All analyses were performed in triplicate and the data are presented in terms of mean \pm standard deviation. Then, the data obtained were analyzed by using one-way Analysis of Variance (ANOVA) (3 treatment) and paired t-test (2 treatment) of Minitab-14.0 software. The comparisons of means were performed by Fisher's Test with confidence level of $\mathrm{p}<0.05$.

\section{Results and discussion}

\subsection{Yield of extracted ASC and PSC from the skin of the} sharpnose stingray (Dasyatis zugei)

Acid soluble collagen (ASC) and pepsin soluble collagen (PSC) were extracted from the skin of the sharpnose stingray and their yield was calculated according to their respective sample weight. There was a significant difference $(p<0.05)$ in terms of yield obtained between ASC and PSC. The yield of extracted PSC $(34.84 \pm 1.26 \%)$ was higher than the extracted ASC $(20.48 \pm 4.41 \%)$.

The differences in yield obtained between extracted ASC and PSC were due to the degree of hydrolysis process during extraction (Liu et al., 2012). The higher yield obtained by PSC as compared to ASC indicates that collagen from the skin of the sharpnose stingray had limited solubility in $0.5 \mathrm{M}$ acetic acid but became more solubilized in the presence of pepsin enzyme. This showed that pepsin treatment offered a higher degree of hydrolysis than acid treatment. This was due to the pepsin enzyme used in extraction tending to cleave the collagen cross-linking specifically at the telopeptide region, leading to higher efficiency (Matmaroh et al., 2011). Moreover, a lower degree of hydrolysis in ASC was due to the presence of intermolecular cross-linking in acid treatment which caused a decrease in solubility of collagen molecule (Matmaroh et al., 2011; Kaewdang et al., 2014). The cross-links in ASC are formed by covalent bonds through the condensation of the aldehyde group with lysine and hydroxylysine at the telopeptide region (Kaewdang et al., 2014).

The higher yield obtained in PSC than that of ASC from the skin of the sharpnose stingray was in agreement with studies conducted on the cartilage of brown-banded bamboo shark with $1.27 \%$ and $9.59 \%$ for ASC and PSC, respectively (Kittiphattanabawon et al., 2010). Nalinanon et al. (2007), obtained similar findings for the skin of bigeye snapper with $5.31 \%$ and $18.7 \%$ for ASC and PSC, respectively. Moreover, according to Kaewdang et al. (2014), the yield of extracted PSC from the swim bladder of yellow-fin tuna $(12.10 \%)$ was 12 fold higher than the yield of ASC extract (1.07\%). This indicates that at a higher degree of hydrolysis, the pepsin will cleave the polypeptide chain at specific region without damaging the integrity of the triple helix structure of collagen, thus lead to a higher yield of PSC (Nalinanon et al., 2010). This finding showed that pepsin treatment offers a higher degree of hydrolysis on the collagen extraction of the sharpnose stingray skin by presenting the higher yield obtained.

\subsection{Chemical compositions of raw material and extracted collagen}

Table 1 shows the chemical compositions of raw skin of sharpnose stingray. The compositions were high in moisture content $(77.99 \pm 0.88 \%)$ followed by protein content $(31.27 \pm 0.44 \%)$, fat content $(1.61 \pm 0.25 \%)$ and an insignificant amount of ash $(0.155 \pm 0.01 \%)$. Similar findings were obtained from red stingray skin, which was found to contain $78 \%$ moisture content and $24.6 \%$ of protein content (Sato et al., 1986). The compositions on raw skin of the sharpnose stingray were also in agreement with the findings by Muyonga et al. (2004) on the skin of Nile perch, where the moisture, protein, fat and ash levels were $72.7 \%, 20.3 \%, 5.0 \%$ and $3.7 \%$, respectively. The findings were also closely similar to those of the chemical composition of skin of ballon fish with the moisture content of $62.23 \%$, followed by

Table 1. Chemical composition on the skin of the sharpnose stingray (Dasyatis zugei) and extracted collagen as compared to commercial collagen

\begin{tabular}{|c|c|c|c|c|c|}
\hline \multirow{2}{*}{ Samples } & \multicolumn{4}{|c|}{ Chemical composition $(\%)$} & \multirow{2}{*}{ Yield (\%) } \\
\hline & Moisture & Protein & Fat & Ash & \\
\hline Raw skin & $77.99 \pm 0.88$ & $31.27 \pm 0.44$ & $1.61 \pm 0.25$ & $0.155 \pm 0.01$ & - \\
\hline ASC & $5.90 \pm 0.19^{\mathrm{a}}$ & $66.62 \pm 2.06^{\mathrm{b}}$ & $0.57 \pm 0.01^{\mathrm{b}}$ & $27.00 \pm 0.83^{b}$ & $20.48 \pm 4.41^{b}$ \\
\hline PSC & $4.69 \pm 0.20^{\mathrm{a}}$ & $50.96 \pm 2.19^{c}$ & $0.25 \pm 0.02^{\mathrm{b}}$ & $30.00 \pm 1.90^{\mathrm{a}}$ & $34.84 \pm 1.26^{\mathrm{a}}$ \\
\hline Commercial collagen & $5.55 \pm 0.50^{\mathrm{a}}$ & $80.00 \pm 10.71^{\mathrm{a}}$ & $11.53 \pm 0.62^{\mathrm{a}}$ & $2.44 \pm 0.56^{\mathrm{c}}$ & - \\
\hline
\end{tabular}

ASC, acid soluble collagen and PSC, pepsin soluble collagen.

Values are expressed as means \pm standard deviation. Values with different superscript within the column are significantly different $(\mathrm{p}<0.05)$. 
protein, fat and ash content with $21.95 \%, 15.87 \%$ and $0.73 \%$, respectively (Huang et al., 2011). The high protein content in the raw skin of the sharpnose stingray indicates strong potential for collagen extraction.

There were no significant differences in moisture content between extracted ASC $(5.90 \pm 0.19 \%)$, PSC $(4.69 \pm 0.20 \%)$ and commercial collagen $(5.55 \pm 0.50 \%)$ $(\mathrm{p}>0.05)$ (Table 1). The significant reduction in moisture content as compared to raw sample was due to the lyophilizing process of collagen prior to analysis, which removed a great mass of water ( $\mathrm{Li}$ et al., 2013). The moisture content of extracted ASC and PSC from the skin of the sharpnose stingray and commercial collagen was in agreement with a study by Wu et al. (2019), in terms of the moisture content of extracted ASC and PSC from scales of red lip croaker, at $5.63 \pm 0.73 \%$ and $4.02 \pm 0.70 \%$, respectively. Similar findings were also obtained by Kittiphattanabawon et al. (2010), for extracted ASC and PSC from the cartilage of brownbanded bamboo shark at $6.54 \pm 0.36 \%$ and $6.62 \pm 0.79 \%$, respectively.

In addition, there were significant differences in protein content between extracted ASC (66.62 $\pm 2.06 \%)$, PSC $(50.96 \pm 2.19 \%)(\mathrm{p}<0.05)$ and commercial collagen $(80.00 \pm 10.71 \%)$ were significantly higher than that of extracted ASC and PSC $(\mathrm{p}<0.05)($ Table 1$)$. The protein content of extracted ASC and PSC from the skin of the sharpnose stingray was lower as compared to the study by Wu et al. (2019) on the extracted ASC and PSC from the scales of the croceine croaker with the protein content of $93.56 \pm 1.86 \%$ and $94.66 \pm 1.89 \%$, respectively. However, the protein levels of commercial collagen were slightly higher as compared to the studies by Hukmi and Sarbon (2017) with the extracted ASC and PSC from the skin of silver catfish having protein contents of $94.21 \pm 0.08 \%$ and $88.25 \pm 0.09 \%$, respectively.

Furthermore, there were no significant differences in fat content between extracted ASC $(0.57 \pm 0.01 \%)$ and PSC $(0.25 \pm 0.02 \%)(p>0.05)$. However, the fat contents of both extracted ASC and PSC from the skin of the sharpnose stingray were significantly lower than those of commercial collagen $(11.53 \pm 0.62 \%)(p<0.05)$ (Table 1). High-fat content in commercial collagen might be due to the absence of defatting before the extraction process. The fat content of extracted ASC and PSC from the skin of the sharpnose stingray was in agreement with findings by $\mathrm{Wu}$ et al. (2019), on the scales of croceine croaker with a fat content of $0.43 \pm 0.15 \%$ and $0.15 \pm 0.08 \%$ for ASC and PSC, respectively. Similar finding on the fat content of ASC was also recorded by Chi et al. (2013) on the cartilage of skate with $0.66 \pm 0.17 \%$.

Furthermore, there were significant differences in ash content between extracted ASC $(27.00 \pm 0.83 \%)$, PSC $(30.00 \pm 1.90 \%)$ and commercial collagen $(2.44 \pm 0.56 \%)$ $(\mathrm{p}<0.05)$ (Table 1). The ash content of extracted ASC and PSC from the skin of sharpnose stingray was higher than acid soluble collagen extracted from the cartilage of red stingray $(14.60 \pm 0.27 \%)$ as studied by $\mathrm{Chi}$ et al. (2013). Ash contents of extracted ASC and PSC were also higher than those of extracted collagen from the cartilage of blacktip shark, with ash content of $0.76 \pm 0.10 \%$ and $0.70 \pm 0.06 \%$ for ASC and PSC, respectively. The high ash content of extracted ASC and PSC might be due to the incomplete removal of minerals as a result of the salting-out process and also the demineralizing steps in pre-treatment.

\subsection{Viscosity analysis}

Figure 1 shows the relative viscosity of extracted acid soluble collagen (ASC) and pepsin soluble collagen (PSC) from the skin of the sharpnose stingray and commercial collagen with a temperature range from 4 to $50^{\circ} \mathrm{C}$. As shown in the figure, the relative viscosity of extracted collagen (ASC and PSC) and commercial collagen decrease with increasing temperature. However, there were no significant differences between extracted ASC and PSC in terms of their relative viscosity trends $(p>0.05)$. The viscosity of both extracted ASC and PSC was slightly decreased during the heating process from 4 to $16^{\circ} \mathrm{C}$, however, drastically decreased in viscosity was observed when it was heated from $16^{\circ} \mathrm{C}$ to $32^{\circ} \mathrm{C}$ and remained constantly low viscosity when heated above $32^{\circ} \mathrm{C}$. Meanwhile, the relative viscosity of commercial collagen decreased gradually during heating from 4 to $40^{\circ} \mathrm{C}$ and remained constant when heated above $40^{\circ} \mathrm{C}$.

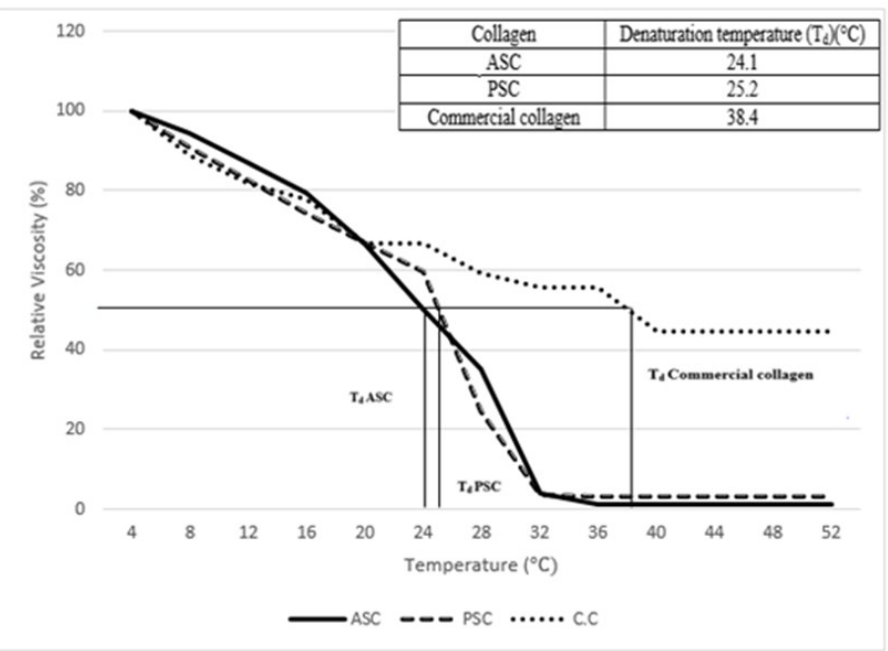

Figure 1. Relative viscosity of ASC, PSC and commercial collagen in temperature range from $4{ }^{\circ} \mathrm{C}$ to $52^{\circ} \mathrm{C}$

The decreases in relative viscosity of extracted and commercial collagen with the temperature increase were due to the heat energy gradually breaking the hydrogen bonds of collagen molecule and lead to the conversion of 
collagen triple helix structure into random coil configurations through a process of thermal depolymerization (Wang et al., 2009). In addition, the constant viscosities of extracted ASC and PSC recorded when heating above $32^{\circ} \mathrm{C}$ and $40^{\circ} \mathrm{C}$ for commercial collagen were due to the complete uncoiling of the collagen protein structure. A collagen molecule will be fully denatured when heated above the denaturation temperature (Duan et al., 2009). The breaking down of the collagen structure will lead to several physical changes, including changes in viscosity, sedimentation, diffusion, light scattering and their optical activity (Wang et al., 2009).

The viscosity trends exhibited by extracted ASC and PSC from the skin of the sharpnose stingray were in agreement with Huang et al. (2011), in which they found that there were no significant differences between their viscosity trends by ASC and PSC of the balloon fish skin. A gradual decrease in viscosity was observed at temperatures ranging from $15^{\circ} \mathrm{C}$ to $40^{\circ} \mathrm{C}$, while at temperatures above $40^{\circ} \mathrm{C}$, constant viscosity was observed (Huang et al., 2011). This indicates that at above denaturation temperature $\left(\mathrm{T}_{\mathrm{d}}\right)$, the complete destruction of intermolecular bond in extracted ASC and PSC leads to a constant viscosity (Kaewdang et al., 2014).

\subsection{Thermal denaturation temperature $\left(T_{d}\right)$}

Denaturation temperature $\left(\mathrm{T}_{\mathrm{d}}\right)$ of extracted collagen was an indication of the degree of changes of protein molecular structure, chemical and physical properties upon heat treatment. Figure 1 shows the denaturation temperature of the extracted ASC and PSC of sharpnose stingray. There were no significant differences in denaturation temperature $\left(\mathrm{T}_{\mathrm{d}}\right)$ between extracted ASC $\left(24.1^{\circ} \mathrm{C}\right)$ and PSC $\left(25.2^{\circ} \mathrm{C}\right)(\mathrm{p}>0.05)$. This showed that acid hydrolysis and enzyme hydrolysis method in isolating ASC and PSC, respectively from the skin of the sharpnose stingray will not affect their relative viscosity as well as denaturation temperature. A similar finding was obtained by Huang et al. (2011), in which no significant difference on denaturation temperature between extracted ASC and PSC from the skin of balloon fish with $29.01^{\circ} \mathrm{C}$ and $30.0^{\circ} \mathrm{C}$, respectively. Thus, cleavage at specific telopeptide region by pepsin treatment had no adverse and significant effect on collagen structure of extracted PSC (Duan et al., 2009).

The results obtained in this study were lower than the denaturation temperature of extracted ASC from carp waste material $\left(28^{\circ} \mathrm{C}\right)$ (Duan et al., 2009). In addition, extracted collagen from land-based organisms such as porcine skin $\left(37^{\circ} \mathrm{C}\right)$ and calfskin $\left(40.8^{\circ} \mathrm{C}\right)$ had higher denaturation as compared to extracted collagen from marine organisms (Liu et al., 2015). This was correlated with their imino acid content (proline and hydroxyproline) of extracted collagen. A higher imino acid content will provide a more stable helical structure and thus a higher denaturation temperature ( $\mathrm{Wu}$ et al., 2014). Moreover, the imino acid content of extracted collagen was found to be correlated to respective body temperature (Duan et al., 2009). According to Liu et al. (2015), the body temperatures of the sources of extracted collagen were directly proportional to their imino acid contents. Conversely, the denaturation temperature of commercial collagen $\left(38.4^{\circ} \mathrm{C}\right)$ was significantly higher than both the extracted collagen $(p<0.05)$. Thus, the higher denaturation temperature of commercial collagen over those of extracted ASC and PSC from the skin of the sharpnose stingray may due to its higher imino acid content.

\subsection{Thermal stability}

Table 2 presents the thermal stability of extracted ASC and PSC from the skin of the sharpnose stingray expressed in terms of maximum temperature $\left(\mathrm{T}_{\max }\right)$ and enthalpy $(\Delta \mathrm{H})$ value. There were no significant differences in maximum temperature $\left(\mathrm{T}_{\max }\right)$ between extracted ASC and PSC ( $>00.05)$. As shown in Table 2, the maximum temperature of extracted ASC was $31.94 \pm 0.13^{\circ} \mathrm{C}$ while for PSC was $31.79 \pm 0.23^{\circ} \mathrm{C}$ $(p>0.05)$. However, there were significant differences in the enthalpy between extracted ASC $(0.59 \pm 0.06 \mathrm{~J} / \mathrm{g})$ and $\operatorname{PSC}(0.24 \pm 0.07 \mathrm{~J} / \mathrm{g})(\mathrm{p}<0.05)$.

Table 2. Maximum temperature and enthalpy of ASC and PSC

\begin{tabular}{ccc}
\hline Collagen & $\mathrm{T}_{\max }\left({ }^{\circ} \mathrm{C}\right)$ & Enthalpy $(\mathrm{J} / \mathrm{g})$ \\
\hline ASC & $31.94 \pm 0.13^{\mathrm{a}}$ & $0.59 \pm 0.062^{\mathrm{a}}$ \\
PSC & $31.76 \pm 0.19^{\mathrm{a}}$ & $0.24 \pm 0.004^{\mathrm{b}}$ \\
\hline
\end{tabular}

ASC, acid soluble collagen and PSC, pepsin soluble collagen. Values are expressed as means \pm standard deviation. Values with different superscript within the column are significantly different $(\mathrm{p}<0.05)$.

According to El-Rashidy et al. (2015), the thermal stability of collagen was positively correlated to that of the amino acid content of collagen. An increase in amino acids content typically imino acids, proline and hydroxyproline would increase the stability of protein helix structure through facilitates the intra-intermolecular cross-linking (Nalinanon et al., 2011). The presence of the hydroxyl group in hydroxyproline would lead to the formation of hydrogen bonding which strengthens collagen helical structure (Kittiphattanabawon et al., 2010). The difference in enthalpy between extracted ASC $(0.59 \pm 0.06 \mathrm{~J} / \mathrm{g})$ and PSC $(0.24 \pm 0.07 \mathrm{~J} / \mathrm{g})$ indicates that pepsin treatment destroyed the collagen structure, while the higher amino 
acid content level in extracted ASC lead to a stronger helical structure. Conversely, higher thermal energy was required to break the intra-molecular bonding in the extracted ASC structure, leading to a higher enthalpy value.

Similar findings were obtained by Kaewdang et al. (2014) on the thermal stability of extracted ASC and PSC from the swim bladder of yellowfin tuna. They found that there were no significant differences between extracted ASC and PSC with a maximum temperature of $32.97 \pm 0.629$ and $33.92 \pm 0.126^{\circ} \mathrm{C}$, respectively but the enthalpy of extracted ASC $(1.786 \pm 0.034 \mathrm{~J} / \mathrm{g})$ was higher than that of extracted PSC $(0.354 \pm 0.056 \mathrm{~J} / \mathrm{g})$. Findings on maximum temperature $\left(\mathrm{T}_{\max }\right)$ of the extracted ASC and PSC were in accordance with viscosity measurements (Figure 1), as pepsin treatment showed no influence on the denaturation temperature of the extracted collagen.

\subsection{Structural properties}

Table 3 shows the structural properties of extracted ASC and PSC from the skin of the sharpnose stingray skin and commercial collagen determined using Fourier transform infrared (FTIR). The FTIR spectra of extracted ASC and PSC exhibited the characteristics peaks of Amide A, I, II and III. However, the characteristic peaks of Amide A were absent in commercial collagen. There were no significant differences between both extracted ASC and PSC in terms of wavenumber and mode of vibration $(\mathrm{p}>0.05)$. These findings correspond to morphological analysis (Table 3 ) in which there was no difference observed between the surface morphology of extracted ASC and PSC.

The amide A band showed no significant difference in the wavenumber between extracted ASC $\left(3423.55 \mathrm{~cm}^{-}\right.$ $\left.{ }^{1}\right)$ and PSC $\left(3439.12 \mathrm{~cm}^{-1}\right)$ which associated with N-H stretching vibration. The presence of amide A group showed the existence of hydrogen bonds in the protein structure of extracted ASC and PSC (Kittiphattanabawon et al., 2010). This study is in agreement with a study conducted by Krishnamoorthi et al. (2017). The amide A band of extracted ASC and PSC from the skin of pharaoh cuttlefish were observed at $3448 \mathrm{~cm}^{-1}$ and $3423 \mathrm{~cm}^{-1}$, respectively and was related to $\mathrm{N}-\mathrm{H}$ stretching vibrations. This showed that pepsin treatment had no adverse effect on the protein structure of extracted PSC. These findings correspond to the thermal stability which showed no significant difference between extracted ASC and PSC (Table 2).

The amide I band showed no significant difference between extracted ASC and PSC on amide I band observed at a wavenumber of $1626.81 \mathrm{~cm}^{-1}$ and 1627.17 $\mathrm{cm}^{-1}$, respectively, with $\mathrm{C}=\mathrm{O}$ stretching vibration (Table 3) $(p>0.05)$. However, a significantly higher amide I band was observed in commercial collagen, with a wavenumber of $1651.54 \mathrm{~cm}^{-1}$ According to Payne and Veis (1988), a decrease in molecular order will result in a shift of the amide I band to a lower wavenumber. Thus, similar molecular order was found in both extracted ASC and PSC but was lower than that of commercial collagen. Amide I wavenumber on extracted ASC and PSC from the skin of sharpnose stingray were slightly lower than ASC $\left(1643 \mathrm{~cm}^{-1}\right)$ and PSC $\left(1648 \mathrm{~cm}^{-1}\right)$ from the swim bladder of yellow-fin tuna (Kaewdang et al., 2014).

The amide II band showed no significant difference between extracted ASC (1552.93 $\left.\mathrm{cm}^{-1}\right)$, PSC (1553.05 $\mathrm{cm}^{-1}$ ) from the skin of the sharpnose stingray and commercial collagen $\left(1543.90 \mathrm{~cm}^{-1}\right)$ with $\mathrm{N}-\mathrm{H}$ bending vibration $(p>0.05)$ (Table 3$)$. The wavenumber of amide II is related to the molecular order of the extracted collagen (Wang et al., 2009). Thus, different extraction methods used in the isolation of ASC and PSC from sharpnose stingray had no adverse effect on the strength of the collagen structure. This finding corresponds to the thermal stability that showed no significant difference between extracted ASC and PSC in terms of thermal denaturation temperature $\left(T_{d}\right)$ and maximum temperature $\left(\mathrm{T}_{\max }\right)$. Amide I wavenumber on extracted ASC and PSC from the skin of sharpnose stingray were in agreement with findings on extracted ASC $\left(1562 \mathrm{~cm}^{-1}\right)$ and PSC $\left(1557 \mathrm{~cm}^{-1}\right)$ of pharaoh cuttlefish skin (Krishnamoorthi et al., 2017).

The amide III band showed no significant difference between extracted ASC $\left(1239.33 \mathrm{~cm}^{-1}\right)$ and PSC

Table 3. Major functional group found in extracted ASC, PSC and commercial collagen

\begin{tabular}{ccccc}
\hline \multirow{2}{*}{ Functional group } & \multirow{2}{*}{ Mode of Vibration } & \multicolumn{3}{c}{ Wavelength $\left(\mathrm{cm}^{-1}\right)$} \\
\cline { 3 - 5 } & & ASC & PSC & Commercial collagen \\
\hline Amide A & N-H stretching & $3423.55 \pm 0.31^{\mathrm{a}}$ & $3439.12 \pm 5.42^{\mathrm{a}}$ & - \\
Amide I & C=O stretching & $1626.81 \pm 3.49^{\mathrm{a}}$ & $1627.17 \pm 3.34^{\mathrm{a}}$ & $1651.54 \pm 5.41^{\mathrm{b}}$ \\
Amide II & N-H bending & $1552.93 \pm 9.43^{\mathrm{a}}$ & $1553.05 \pm 9.63^{\mathrm{a}}$ & $1543.90 \pm 3.61^{\mathrm{a}}$ \\
Amide III & N-H bending & $1239.33 \pm 0.87^{\mathrm{a}}$ & $1238.81 \pm 0.00^{\mathrm{a}}$ & $1242.67 \pm 0.76^{\mathrm{b}}$ \\
\hline
\end{tabular}

ASC, acid soluble collagen and PSC, pepsin soluble collagen.

Values are expressed as means \pm standard deviation. Values with different superscript within the column are significantly different $(\mathrm{p}<0.05)$. 
$\left(1238.81 \mathrm{~cm}^{-1}\right)$ from the skin of the sharpnose stingray with N-H bending vibration $(p>0.05)$ (Table 3$)$. However, the wavenumber of amide III band (1242.67 $\mathrm{cm}^{-1}$ ) on commercial collagen were significantly higher than extracted ASC and PSC $(p<0.05)$. The presence of the amide III band indicated that the triple helical structure was present in collagen (Pati et al., 2010). This indicated that the triple helix structure was present in extracted ASC and PSC and commercial collagen. Thus, this had proved that the extraction method would not affect the extracted ASC and PSC from the skin of the sharpnose stingray on their protein structure. The findings of amide III band wavenumber on extracted ASC and PSC were in agreement with studies by Matmaroh et al. (2011), which there were no significant differences between the wavenumber of amide III band on extracted ASC $\left(1239 \mathrm{~cm}^{-1}\right)$ and PSC $\left(1236 \mathrm{~cm}^{-1}\right)$ from the swim bladder of calta.

\subsection{Solubility}

Figure 2 shows the effect of $\mathrm{pH}$ from the range of 1 to 10 on the solubility of extracted acid soluble collagen (ASC) and pepsin soluble collagen (PSC) from the skin of the sharpnose stingray and commercial collagen. The protein solubility is an important functional property which provides a prediction of the potential application of collagen. According to Figure 2, ASC showed higher solubility in acidic condition at $\mathrm{pH}$ range from 1 to 6 and exhibited maximum solubility at $\mathrm{pH} 2$. The lowest solubility of extracted ASC was observed at $\mathrm{pH} 7$ followed by a slight increase in solubility at basic $\mathrm{pH}$. A closely similar trend was observed for extracted PSC which higher solubility in acidic condition at $\mathrm{pH}$ range from 1 to 5 and exhibit maximum solubility at $\mathrm{pH} 4$ while a decrease in solubility at $\mathrm{pH}>5$. Similarly, to the ASC, the lowest solubility of PSC was at neutral $\mathrm{pH}$ and a slightly gradual increase at basic $\mathrm{pH}>7$ was observed. The increase in solubility in basic condition was due to the repulsion forces between polypeptide chains as the $\mathrm{pH}$ was higher than isoelectric points (Nalinanon et al., 2011). Meanwhile, the solubility of commercial collagen exhibited a large variation as compared to that of extracted ASC and PSC from sharpnose stingray. The highest solubility was recorded in strong acidic condition ( $\mathrm{pH}$ 1), weak acidic condition ( $\mathrm{pH} \mathrm{6)}$ and strong alkali condition ( $\mathrm{pH} 10)$. The differences in solubility between commercial collagen and the collagen extracted from sharpnose stingray may be caused by the differences in their molecular properties and conformations.

Both extracted ASC and PSC from the skin of the sharpnose stingray showed the highest solubility at different optimal $\mathrm{pH}$ (Figure 2). The maximum solubility of extracted PSC was at $\mathrm{pH} 4$ which was more basic than the maximum solubility of extracted ASC from sharpnose stingray skin with the highest solubility at $\mathrm{pH}$ 2. This was in agreement with Kaewdang et al. (2014), who found that the highest solubility of PSC was at a higher $\mathrm{pH}$ value than that of ASC. This phenomenon was due to the alteration of isoelectric point (pI) by the pepsin treatment (Kaewdang et al., 2014). Pepsin hydrolyzed collagen through the cutting at the specific part on telopeptide regions of the collagen polypeptide chain (Yang and Shu, 2014). Thus, this indicated that pepsin treatment gave a significant effect on the solubility of extracted PSC.

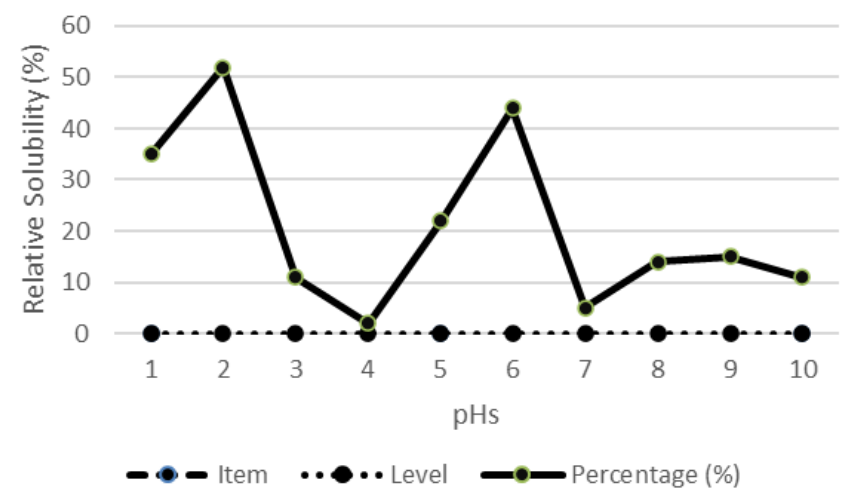

Figure 2. Solubility of extracted ASC, PSC and commercial collagen in $\mathrm{pH}$ range of 1 to 10

In addition, both ASC and PSC from the skin of the sharpnose stingray exhibited the lowest solubility at neutral $\mathrm{pH}(\mathrm{pH}$ 7). A decrease in solubility of extracted collagen at particular $\mathrm{pH}$ was due to the hydrophobic interaction among collagen molecule and the total net charge of protein molecules becomes zero at their isoelectric points (pI) (Chi et al., 2013). When extracted collagen was at the isoelectric point (pI), the increase in hydrophobic-hydrophobic interaction will lead to the lowest solubility which the collagen tends to precipitate and aggregate (Chi et al., 2013). This indicates that both the extracted ASC and PSC had isoelectric points at $\mathrm{pH}$ 7. Thus, the similar isoelectric points exhibited between extracted ASC and PSC showed that isoelectric point (pI) of extracted collagen will not be affected by different extraction method. The findings on the solubility of extracted ASC and PSC from the skin of the sharpnose stingray was in agreement with findings recorded by Chi et al. (2013), who extracted ASC from the cartilage of red stingray with maximum solubility at $\mathrm{pH} 2$ and was soluble in an acidic $\mathrm{pH}$ ranging from 1 to 4. Minimum solubility was recorded at $\mathrm{pH} 7$, followed by a slightly increased insolubility when $\mathrm{pH}$ increased from 7 to 11. This indicates that the removal of telopeptide regions by pepsin treatment affects the protonation or deprotonation of charged amino and carboxyl group, leading to alteration of solubility properties of extracted collagen (Jongjareonrak et al., 
2005).

\subsection{Morphological properties}

Figure 3 shows the microstructure of both extracted ASC and PSC from the skin of sharpnose stingray. Both the extracted ASC and PSC were loose and possessed slender, less uniform and irregular fibrous network structures, with large and irregular pores observed between the fibrils. The formation of large pore size was due to the evaporation of fluids from the collagen surface (Chi et al., 2013; Veeruraj et al., 2013). According to Veeruraj et al. (2013), the shape and wall morphology of pores and the interconnectivity of collagen are important parameters for biomaterials. In addition, agglomerate particles were also observed on the surface of both extracted ASC and PSC which is the residue salt remained after the salt-out process (Ramirez-Leal et al., 2009).

Surface morphology of extracted ASC and PSC from the skin of the sharpnose stingray was in agreement with the surface morphology of extracted ASC from the cartilages of scalloped hammerhead, red stingray and skate (Chi et al., 2013). In addition, Veeruraj et al. (2013), found that both the extracted ASC and PSC from the skin of eel fish possessed fibril and porous structure in three-dimensional forms. Moreover, large pore sizes were also observed in extracted ASC and PSC from silver catfish skin which showed flaky and porous structure (Hukmi and Sarbon, 2017). The similar surface morphology observed on extracted collagen corresponds to the structural properties which showed no significant difference in terms of wavenumber for the functional group, Amide A, I, II and III and thermal stability (Table 3 ), which showed no significant difference in maximum temperature between extracted ASC and PSC from the skin of sharpnose stingray.
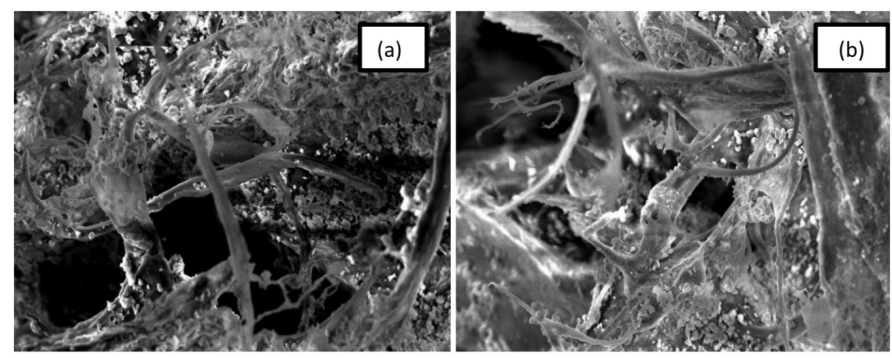

Figure 3. Surface morphology of (a) ASC and (b) PSC extracted from the skin of sharpnose stingray

\section{Conclusion}

Overall, the enzyme hydrolysis method using pepsin was preferable to isolate pepsin soluble collagen (PSC) from the skin of the sharpnose stingray due to its higher efficiency in terms of yield and superior physicochemical properties. Both extracted ASC and
PSC were highest in protein content followed by ash, moisture and fat content. In addition, the extraction method had no significant effect on the thermal stability of extracted ASC and PSC which correspond to the surface morphology and structural properties. The higher enthalpy exhibited by ASC was due to the destruction of the PSC structure by pepsin treatment and higher amino acid content in ASC. Furthermore, both extracted ASC and PSC showed high solubility at acidic $\mathrm{pH}$ but are soluble at different optimal $\mathrm{pH}$ due to the alteration of isoelectric point $(\mathrm{pI})$ by the pepsin treatment.

\section{Conflict of interest}

The authors declare no conflict of interest.

\section{References}

AOAC. (2000). Official Methods of analysis. Arlington: Association of Official Chemist Corporation.

Baderi, N.A. and Sarbon, N.M. (2019). Microstructure, extractability and physicochemical properties of shortfin scad (Decapterus macrosoma) bone collagen as influenced by acetic acid concentration. International Food Research Journal, 26(2), 451 458.

Chi, C.F., Wang, B., Li, Z.R., Luo, H.Y. and Ding, G.F. (2013). Characterization of acid- soluble collagens from the cartilages of scalloped hammerhead (Sphyrna lewini), red stingray (Dasyatis akajei), and skate (Raja porosa). Food Science and Biotechnology, 22(4), 909-916. https:// doi.org/10.1007/s10068-013-0163-0

Duan, R., Zhang, J., Du, X., Yao, X. and Konno, K. (2009). Properties of collagen from skin, scale and bone of carp (Cyprinus carpio). Food Chemistry, 112(3), 702-706. https://doi.org/10.1016/ j.foodchem.2008.06.020

El-Rashidy, A.A., Gad, A., Abu-Hussein, A.E.H.G., Habib, S.I., Badr, N.A. and Hashem, A.A. (2015). Chemical and biological evaluation of Egyptian Nile Tilapia (Oreochromis niloticas) fish scale collagen. International Journal of Biological Macromolecules, 79, 618-626. https:// doi.org/10.1016/j.ijbiomac.2015.05.019

Hamdan, F.S. and Sarbon, N.M. (2019). Isolation and characterization of collagen from Fringescale sardinella (Sardinella fimbriata) waste materials. International Food Research Journal, 26(1), 133140.

Huang, Y.R., Shiau, C.Y., Chen, H.H. and Huang, B.C. (2011). Isolation and characterization of acid and pepsin-solubilized collagens from the skin of balloon fish (Diodon holocanthus). Food Hydrocolloids, 25 
(6), 1507-1513.

https://doi.org/10.1016/

j.foodhyd.2011.02.011

Hukmi, N. and Sarbon, N.M. (2017). Isolation and characterization of acid soluble collagen (ASC) and pepsin soluble collagen (PSC) extracted from silver catfish (Pangasius s.p) skin. International Food Research Journal, 25(5), 1785-1791.

Jongjareonrak, A., Benjakul, S., Visessanguan, W. and Tanaka, M. (2005). Isolation and characterization of collagen from bigeye snapper (Priacanthus macracanthus) skin. Journal of the Science of Food and Agriculture, 85(7), 1203-1210. https:// doi.org/10.1002/jsfa.2072

Kaewdang, O., Benjakul, S., Kaewmanee, T. and Kishimura, H. (2014). Characteristics of collagens from the swim bladders of yellowfin tuna (Thunnus albacares). Food Chemistry, 155, 155, 264-270. https://doi.org/10.1016/j.foodchem.2014.01.076

Kittiphattanabawon, P., Benjakul, S., Visessanguan, W. and Shahidi, F. (2010). Isolation and characterization of collagen from the cartilages of brown banded bamboo shark (Chiloscyllium punctatum) and blacktip shark (Carcharhinus limbatus). LWT-Food Science and Technology, 43(5), 792-800. https:// doi.org/10.1016/j.lwt.2010.01.006

Krishnamoorthi, J., Ramasamy, P., Shanmugam, V. and Shanmugam, A. (2017). Isolation and partial characterization of collagen from outer skin of Sepia pharaonis (Ehrenberg, 1831) from Puducherry coast. Biochemistry and Biophysics Reports, 10, 3945. https://doi.org/10.1016/j.bbrep.2017.02.006

Li, Z.R., Wang, B., Chi, C.F., Zhang, Q.H., Gong, Y.D., Tang, J.J. and Ding, G.F. (2013). Isolation and characterization of acid soluble collagens and pepsin soluble collagens from the skin and bone of Spanish mackerel (Scomberomorous niphonius). Food Hydrocolloids, 31(1), 103-113. https:// doi.org/10.1016/j.foodhyd.2012.10.001

Lim, K.C., Chong, V.C., Lim, P.E. and Yurimoto, T. (2014). Length-weight relationship of stingrays in Kuala Selangor, Malaysia. Journal of Applied Ichthyology, 30(5), 1096-1098. https:// doi.org/10.1111/jai.12455

Liu, D. Liang, L. Regenstein, J.M. and Zhou, P. (2012). Extraction and characterisation of pepsin-solubilised collagen from fins, scales, skins, bones and swim bladders of bighead carp (Hypophthalmichthys nobilis). Food Chemistry, 133(4), 1441-1448. https://doi.org/10.1016/j.foodchem.2012.02.032

Liu, D., Wei, G., Li, T., Hu, J., Lu, N., Regenstein, J.M. and Zhou, P. (2015). Effects of alkaline pretreatments and acid extraction conditions on the acid-soluble collagen from grass carp (Ctenopharyngodon idella) skin. Food Chemistry, 172, 836-843. https://doi.org/10.1016/ j.foodchem.2014.09.147

Lowry, C.C., Kraeft, N.H. and Hughes, F.A. (1951). Blastomycosis of the lung. The American Journal of Surgery, 81(6), 676-679. https:// doi.org/10.1016/0002-9610(51)90161-4

Matmaroh, K., Benjakul, S., Prodpran, T., Encarnacion, A.B. and Kishimura, H. (2011). Characteristics of acid soluble collagen and pepsin soluble collagen from scale of spotted golden goatfish (Parupeneus heptacanthus). Food Chemistry, 129(3), 1179-1186. https://doi.org/10.1016/j.foodchem.2011.05.099

Matsunuma, M., Motomura, H., Matsuura, K., Shazili, N.A.M. and Ambak, M.A. (2011). Fishes of Terengganu - East Coast of Malay Peninsula, Malaysia. Malaysia: National Museum of Nature and Science, Universiti Malaysia Terengganu and Kagoshima University Museum.

Muyonga, J.H., Cole, C.G.B. and Duodu, K.G. (2004). Characterization of acid soluble collagen from skins of young and adult Nile perch (Lates niloticus). Food Chemistry, 85(1), 81-89. https:// doi.org/10.1016/j.foodchem.2003.06.006

Nalinanon, S., Benjakul, S., Visessanguan, W. and Kishimura, H. (2007). Use of pepsin for collagen extraction from the skin of bigeye snapper (Priacanthus tayenus). Food Chemistry, 104(2), 593 $-601$. https://doi.org/10.1016/ j.foodchem.2006.12.035

Nalinanon, S., Benjakul, S. and Kishimura, H. (2010). Collagens from the skin of arabesque greenling (Pleurogrammus azonus) solubilized with the aid of acetic acid and pepsin from albacore tuna (Thunnus alalunga) stomach. Journal of the Science of Food and Agriculture, 90(9), 1492-1500. https:// doi.org/10.1002/jsfa.3973

Nalinanon, S., Benjakul, S., Kishimura, H. and Osako, K. (2011). Type I collagen from the skin of ornate threadfin bream (Nemipterus hexodon): Characteristics and effect of pepsin hydrolysis. Food Chemistry, 125(2), 500-507. https://doi.org/10.1016/ j.foodchem.2010.09.040

Pal, G.K. and Suresh, P.V. (2017). Physico-chemical characteristics and fibril-forming capacity of carp swim bladder collagens and exploration of their potential bioactive peptides by in silico approaches. International Journal of Biological Macromolecules, 101, 304-313. https:// doi.org/10.1016/j.ijbiomac.2017.03.061

Pati, F., Adhikari, B. and Dhara, S. (2010). Isolation and 
characterization of fish scale collagen of higher thermal stability. Bioresource Technology, 101(10), 3737-3742. j.biortech.2009.12.133

https://doi.org/10.1016/

Payne, K.J. and Veis, A. (1988). Fourier transform IR spectroscopy of collagen and gelatin solutions: deconvolution of the amide I band for conformational studies. Biopolymers, 27 (11), 17491760. https://doi.org/10.1002/bip.360271105

Ramirez-Leal, R., Esparza-Ponce, H.E., Varela-Sortillón, A., Astorga-Reyes, A. and Roman-B, A. (2009). Elemental Chemical Composition, Size and Morphological Characterization of Individual Atmospheric Particles within an Air Quality Program. Microscopy and Microanalysis, 15(2), 1300. https://doi.org/10.1017/S1431927609095841

Sato, K., Yoshinaka, R., Sato, M. and Ikeda, S. (1986). A simplified method for determining collagen in fish muscle. Bulletin of the Japanese Society of Scientific Fisheries, 52(5), 889-893. https://doi.org/10.2331/ suisan.52.889

Schmidt, M.M., Dornelles, R.C.P., Mello, R.O., Kubota, E.H., Mazutti, M.A., Kempka, A.P. and Demiate, I.M. (2016). Collagen extraction process. International Food Research Journal, 23 (3), 913-922.

Silvipriya, K. S., Krishna Kumar, K., Bhat, A.R., Dinesh Kumar, B., John, A. and Lakshmanan, P. (2015). Collagen: Animal sources and biomedical application. Journal of Applied Pharmaceutical Science, 5(3), 123-127. https://doi.org/10.7324/ JAPS.2015.50322

Veeruraj, A., Arumugam, M. and Balasubramanian, T. (2013). Isolation and characterization of thermostable collagen from the marine eel-fish (Evenchelys macrura). Process Biochemistry, 48 (10), 1592-1602. https://doi.org/10.1016/ j.procbio.2013.07.011

Wang, L., An, X., Yang, F., Xin, Z., Zhao, L. and Hu, Q. (2009). Isolation and characterization of collagens from the skin, scale and bone of deep-sea redfish (Sebastes mentella). Food Chemistry, 108(2), 616623. https://doi.org/10.1016/j.foodchem.2007.11.017

Wu, G.P., Wang, X.M., Lin, L.P., Chen, S.H. and Wu, Q.Q. (2014). Isolation and characterization of pepsin -solubilized collagen from the skin of black carp (Mylopharyngdon piceus). Advances in Bioscience and Biotechnology, 5(7), 642-650. https:// doi.org/10.4236/abb.2014.57076

Wu, J., Kong, L., Zhang, J. and Chen, W. (2019). Extraction and Properties of Acid-Soluble Collagen and Pepsin-Soluble Collagen from Silver Carp
(Hypophthalmichthys molitrix) Scales: Prerequisite Information for Fishery Processing Waste Reuse. Polish Journal of Environmental Studies, 28(4), 2923-2930. https://doi.org/10.15244/pjoes/93742

Yang, H. and Shu Z.B. (2014). The extraction of collagen protein from pigskin Journal of Chemical and Pharmaceutical Research, 6(2), 683-687. 